\title{
What is theoretical progress of science?
}

\author{
Juha Saatsi ${ }^{1}$
}

Received: 2 August 2015 / Accepted: 1 May 2016 / Published online: 19 May 2016

(C) The Author(s) 2016. This article is published with open access at Springerlink.com

\begin{abstract}
The epistemic conception of scientific progress equates progress with accumulation of scientific knowledge. I argue that the epistemic conception fails to fully capture scientific progress: theoretical progress, in particular, can transcend scientific knowledge in important ways. Sometimes theoretical progress can be a matter of new theories 'latching better onto unobservable reality' in a way that need not be a matter of new knowledge. Recognising this further dimension of theoretical progress is particularly significant for understanding scientific realism, since realism is naturally construed as the claim that science makes theoretical progress. Some prominent realist positions (regarding fundamental physics, in particular) are best understood in terms of commitment to theoretical progress that cannot be equated with accumulation of scientific knowledge.
\end{abstract}

Keywords Bird $\cdot$ Newton $\cdot$ Scientific knowledge $\cdot$ Scientific progress $\cdot$ Scientific realism

\section{Introduction}

What is scientific progress? A simple question. Or, a question that is simple to state, at least. Bird (2007) thinks the question also has a simple answer: science progresses by cumulating knowledge.

Science (or some particular scientific field or theory) makes progress precisely when it shows the accumulation of scientific knowledge; an episode in science

$\triangle$ Juha Saatsi

J.T.Saatsi@leeds.ac.uk

1 School of Philosophy, Religion, and History of Science, University of Leeds, Leeds, UK 
is progressive when at the end of the episode there is more knowledge than at the beginning. (64)

This sums up the epistemic conception of scientific progress. It is intuitive, straightforward, and has a venerable history. But it is too simple! I will examine and expose its shortcomings specifically in relation to theoretical progress, showing how theoretical progress can in important ways transcend scientific knowledge. Given a close conceptual connection between theoretical progress and scientific realism this has significant repercussions for our understanding of scientific realism, too. ${ }^{1}$

What is theoretical progress in science, more specifically? By 'theoretical' progress, as I use the term, I mean the kind of progress that goes beyond the progress that practically everyone-including instrumentalists or empiricists-associates with science. As such, theoretical progress is naturally characterised, ab initio, in contrastive terms, in relation to various specific ways for science to progress which do not count as 'theoretical' in this sense. For example, one non-theoretical aspect of progress has to do with the improving empirical accuracy of theoretical representations in science. ${ }^{2}$ Although it is not a straightforward matter to say what this kind of progress exactly amounts to, one influential and powerful idea is to think of it in terms of progress towards (complete) empirical adequacy. (van Fraassen 1980) There are also various forms of progress in the 'instrumental' features of science: in addition to the development of scientific instruments, one can think of new mathematical and computational methods, for instance. ${ }^{3}$ There are various examples of such broadly pragmatic progress, corresponding to all kinds of useful cognitive scaffolding, from the development of a powerful notation (e.g. Feynman diagrams), to a heuristically useful mode of presentation (e.g. acceleration in response to fictitious Coriolis force). 'Theoretical progress', as I use the term, denotes scientific progress that is extra-empirical, extra-instrumental, extra-pragmatic. It is whatever progress scientists make (or possibly could make) with theories, going beyond the progress science exhibits in its empirical, instrumental, or pragmatic aspects. Scientific realists maintain that science actually exhibits also theoretical progress.

With theoretical progress thus understood, we can ask: what exactly is it? ${ }^{4}$ What exactly does the realist claim amount to? I will answer these questions by analysing the notion of theoretical progress, aiming to characterise it in a way that respects our

\footnotetext{
${ }^{1}$ Rowbottom (2010) also denies that the epistemic view provides a necessary condition for scientific progress. More specifically, Rowbottom denies that a progress-inducing scientific development must be justified; hence he denies that these developments must be knowledge-inducing, since knowledge entails justification. (Against Bird (2007), Rowbottom (2010, pp. 242-243) also considers the possibility of progress with justified true beliefs that do not amount to knowledge. See also the exchange in Rowbottom (2008) and Bird (2008) on the constitutive vs. instrumental role of justification.) My argument, by contrast, focuses on progressive developments in science, involving false theories, that are justified but nevertheless need not yield accumulation of knowledge sufficient for grounding the progress.

2 'Theoretical representation' is an umbrella term for all types of theories, models, simulations, et cetera, that can be construed as representing the world. From now on I just use 'theory', for short.

${ }^{3}$ See Douglas (2014) for the importance of broadly 'instrumental' progress in increased capacity to intervene, control, and predict the empirical world.

${ }^{4}$ Or, we can ask 'what would count as theoretical progress?', disregarding the realist issue whether science actually makes any theoretical progress.
} 
intuitions and encompasses important senses of extra-empirical and extra-instrumental progress. Correspondingly, as an upshot, I will characterise scientific realism in new ways-with scientific realism construed as claiming that science actually makes some kind of theoretical progress. I will do all this in the context of the epistemic conception of progress, pointing to the limits of this conception in relation to theoretical progress in particular, arguing that it fails to fully capture some strong intuitions about theoretical progress and, correspondingly, about realism. ${ }^{5}$ (Whether or not the epistemic conception of progress fully or naturally captures other aspects of scientific progress does not concern me here.)

The next section presents a natural intuition about theoretical progress, that we want to respect despite its variance with the epistemic conception (reviewed in Sect. 3). The rest of the paper then spells out, refines, and defends this intuition and an associated analysis of scientific realism.

\section{An intuition about theoretical progress}

Intuitively, one undeniable dimension of theoretical progress has to do with how well our theories latch onto unobservable reality: the better contact our theoretical assumptions make with reality, the better. The intuitive idea of 'latching onto' reality is a bit vague and metaphorical. Don't worry; stick with me. I will elucidate it in due course, in Sect. 4.

Intuitive, also, theoretical progress cannot just be a matter of theories making better contact with the unobservable reality, however. We do not make theoretical progress simply by adopting a new theory that latches better onto unobservable reality, since it would not count as theoretical progress if we adopted such a theory on some arbitrary grounds - merely accidentally latching better onto reality. It would not count as progress if such theory somehow emerged as the leading contender by sheer luck-as an outcome of irrational, unreliable pseudo-scientific theorising, say, without appropriate evidential support. (Bird 2007, pp. 66-67) Intuitively, then, theoretical progress is also partly a matter of a theory being suitably supported by evidence.

We can succinctly capture the two intuitions above as follows:

TP Science makes theoretical progress if a theoretical representation that is better supported by scientific evidence latches better onto unobservable reality. ${ }^{6}$

\footnotetext{
5 In appealing to 'our intuitions' about (theoretical) progress I am not claiming or aiming to capture a 'folk' concept of scientific progress (cf. Mizrahi and Buckwalter 2014). Rather, I take 'theoretical progress' to be a term of art in well-established philosophical debates about scientific realism and how scientific theorising relates to reality. In the context of these debates the notion of 'theoretical' progress, and its contrast to e.g. 'empirical' or 'instrumental' progress, serves to delineate positions that philosophers engaged in those debates care about. Our (viz. philosophers') intuitions about (theoretical) progress matter to the extent they can, for example, point towards novel philosophical positions worth investigating and defending in the context of those debates. Also, along with many other authors I view philosophical debates about scientific progress as inherently normative, involving judgments about values or aims of 'good science' that cannot be reduced to the content of folk intuitions about progress (cf. Niiniluoto 2011; Rowbottom 2015).

$6 T P$ only gives a sufficient condition for theoretical progress, leaving it open whether there are other forms of such progress not captured by this characterisation. I'll explain my reasons for leaving it open in this way in Sect. 6.
} 
The rest of this paper spells out, refines, and defends the idea that TP captures a dimension of theoretical progress. There are three things in particular to clarify with respect to $T P$ and scientific realism. It is noteworthy, first of all, that $T P$ does not characterise theoretical progress as an epistemic matter: TP does not speak of theoretical knowledge. One may find this puzzling: isn't the notion of theoretical progress intimately connected to (or even identical to) the notion of accumulating theoretical knowledge? Section Sect. 3 discusses what is right and what is wrong with this reaction. Secondly, we need to clarify what is meant by 'latching better onto' reality (Sect. 4). Finally, having done all that, we need to sharpen our grasp on scientific realism by attending to the connection between theoretical progress and scientific realism (Sect. 5).

\section{Theoretical progress and knowledge}

\subsection{The epistemic conception of progress versus TP}

According to the epistemic conception there is progress in science 'precisely when it shows accumulation of knowledge.' (Bird 2007, p. 64) With this in mind, theoretical progress more specifically is naturally equated with accumulation of theory-mediated knowledge about the unobservable. ${ }^{7}$

The epistemic conception of progress is intuitive and attractive. Also, it is natural to think that theoretical progress, more specifically, should be intimately linked to accumulating scientific knowledge. For isn't theoretical progress pre-theoretically a matter of learning new facts about the unobservable world? Isn't it about getting to know through well-functioning scientific theorising what the unobservable reality is like? And in as far as scientific realism can be construed as standing for the idea that science makes theoretical progress, doesn't the latter notion necessarily incorporate an epistemological aspect, just as realism does? Such intuitions support the equation of progress with accumulation of true scientific beliefs that qualify as knowledge. ${ }^{8}$ It is also suggested by many realist writings, which often thus relate progress to knowledge about the unobservable, as e.g. Leplin (1984, p. 2) does: "What realists do share in common are the convictions that scientific change is, on balance, progressive, and that science makes possible knowledge of the world beyond its accessible, empirical manifestations."

But natural though this equation is, it is too simple. Even if much of scientific progress can be captured as accumulation of some kind of knowledge, I will now argue that it is possible to have theoretical progress that does not amount to accumulation of knowledge. Therefore, a comprehensive conception of progress must be broader than the epistemic conception.

My argument will focus on $T P$. We have already noted that $T P$ does not say anything at all about scientific knowledge, in stark contrast with the epistemic conception of

\footnotetext{
7 This is more or less how Bird understands it, too (2007, p. 79). Many others have in the same vein construed 'cognitive progress' of science in terms of accumulation of knowledge, including also knowledge about the current limits of science, for example.

${ }^{8}$ For a true belief to qualify as knowledge it needs to be epistemically acceptable, which according to the consensus requires a justification of some sort and/or being produced by a reliable mechanism.
} 
progress. According to $T P$, theoretical progress can be a matter of better empirically supported theories latching better onto reality (in a way that is not accidental). I will argue that there need not be accumulation of knowledge to accompany such progress in the way required by the epistemic conception. ${ }^{9}$

Let me begin by offering a purely conceptual reason for thinking that the epistemic conception puts too much weight on knowledge. 'Progress', in general, is a resultoriented, evaluative notion that applies to a positive achievement. In connection with science, our theories can (in principle, at least) represent correctly aspects of unobservable reality, and this can be regarded as a positive achievement (in as as far as it really is an achievement and not a mere happenstance). 'Theoretical progress', then, is naturally regarded as taking place if our theories are doing better in this way-if our theories are getting better at representing the unobservable. But progress thus characterised makes no mention of knowledge; it is therefore not primarily an epistemic matter. (It is an epistemic matter only in the sense that accepted theories must be suitably supported by evidence.)

Take, for example, a constructive empiricist. She can agree both that our theories purportedly represent unobservable reality, and that theories can do better or worse in this respect, even though she takes 'the aim' of science to be the production of empirically adequate theories, and she thinks that we are never in a position to know how well our theories latch onto unobservable reality. An empiricist can admit that, for all we know, science may or may not make theoretical progress (in addition to progress towards empirical adequacy), even though our knowledge about the world is limited to its observable features. ${ }^{10}$ (Or she could say that even if science makes theoretical progress, we are never rationally compelled to believe it.) So, there is a sense in which de facto theoretical progress seems compatible even with constructive empiricism. But this could not be so if the notion of 'theoretical progress' was exhausted by the epistemic conception according to which theoretical progress entails accumulation of theory-mediated knowledge about the unobservable world.

The intelligibility of theoretical progress that thus transcends scientific knowledge indicates that such progress need not be an epistemic matter. Having said that, I think we would do well to hang onto the idea that the notion of theoretical progress is closely connected to scientific realism. We can do this by incorporating into our analysis of theoretical progress a minimal epistemic requirement: it seems reasonable to require that for science to exhibit progress it must be in principle possible to know about the progress made by reflecting on the nature of scientific enquiry, empirical evidence,

\footnotetext{
9 One might think that there is always known empirical progress accompanying $T P$, since the characterisation of $T P$ requires that the new theory has better empirical support than the preceding theories. But this is too quick: better empirical support need not be a matter of increasing empirical adequacy. It can also be a matter of seeing how the same observable phenomena better fit our changing theoretical representations of the unobservable reality. For example, a single consistent theory that captures certain phenomena is empirically better supported than a mutually inconsistent conjunction of two theories that collectively capture the exactly same phenomena.

10 The distinction between observable and unobservable features of the world has been drawn in different ways in the realism debate. For the present discussion nothing much hangs on this, as the kind of theoretical progress I am concerned with has to do with features of the world that are undeniably unobservable (Cf. van Fraassen 2003).
} 
theory-change, etc. This requirement secures the possibility of realism, in case science as a matter of fact is thus progressive, and it is enough to render theoretical progress incompatible with empiricism, if the empiricist denies the possibility of knowing whether there actually is theoretical progress. Still, this notion remains independent of the question whether we actually do know of such progress or not. Whether or not there is theoretical progress still need not be a primarily epistemic matter.

Thinking about 'theoretical progress' as a result-oriented, evaluative notion thus shows how it is only epistemic in a broad sense; it is not primarily concerned with knowledge. Next I will discuss how the epistemic conception of progress furthermore turns out to be unduly restrictive, since we can easily conceive of progressive theoretical developments that actually do not exhibit a pertinent kind of accumulation of knowledge.

\subsection{The epistemic conception and the grounding question}

Since knowledge entails truth, accumulation of scientific knowledge requires accumulation of known truths. I will now argue that the notion of progress must be broader than accumulation of knowledge since we can conceive of ways in which theories can improve as per TP, without a corresponding accumulation of known truths. (Correspondingly, I will argue (in Sect. 5) that the realist idea that science really does exhibit theoretical progress turns out to be broader than the notion that there really is accumulation of theoretical knowledge.)

We can pose the following question-call it the Grounding Question - to the epistemic conception of progress. Since knowledge entails truth, accumulation of scientific knowledge requires accumulation of scientific truths. But where in science can we locate the truths that thus ground theoretical progress? ${ }^{11}$

What makes the Grounding Question pressing is the fact that our theories and their 'components' - however these are construed-rarely attain full truth: they are typically at best 'approximately true', or 'partially true', or 'partially veridical' in some sense. But knowledge is grounded in truth simpliciter. ${ }^{12}$ So a string of questions arises: Where in science are these progress-grounding exact truths to be found? What are those truths about? How are those truths accessible to scientists who (according to our intuitions) make progress? The epistemic conception must answer these questions in a way that respects our intuitions about theoretical progress.

Bird (2007) recognises the need to respond to the Grounding Question. He doesn't consider it in its full generality, but only in connection with his evaluation of the competing 'semantic' conception of progress, according to which progress is a matter of increasing verisimilitude. More specifically, Bird evaluates the following consideration that he moots on behalf of the semantic conception of progress.

\footnotetext{
11 Questions similar to my Grounding Question have been asked about idealised models, for example. See e.g. Elgin (2007), Jones (2013), Mizrahi (2012).

12 The factivity of knowledge - that knowledge entails truth-is almost universally accepted platitude about knowledge. There is, of course, a great deal of disagreement about the exact epistemic relation between knowledge and the relevant truth.
} 
Theories are very often at best only approximately true; they rarely attain full truth. Since knowledge entails full truth, theories cannot be the objects of knowledge. Consequently we cannot be accumulating knowledge in such cases. Hence the cumulative knowledge account cannot explain the sense in which our theories are getting better. The verisimilitude account can explain this and so is a better account. (76)

Although Bird denies that there is a single notion of verisimilitude-informal or otherwise - that could be used to characterise progress in general, he nevertheless argues that insofar as verisimilitude can be meaningfully used to describe closeness to truth in some specific contexts, it provides a bridge to the relevant true propositions that can be taken as objects of knowledge in the epistemic conception.

If $P$ is approximately true, then the proposition $Q$, that $P$ is approximately true, is itself true, not merely close to the truth. This is legitimate, since if 'planets travel in ellipses' is a scientific proposition, then so is 'approximately, planets travel in ellipses'. Even if $P$ is not true and so not knowable, $Q$ ( $Q=$ approximately $P$ ) might well be knowable. (76)

Bird thus moves from an approximately true proposition (or theory) to a fully true proposition concerning the approximation. Call this Bird's move. It is a natural move to make; what should we make of it? It is critical to recognise that while it is undeniably effective move to make in relation to some strictly false propositions (e.g. 'planets travel in ellipses'), it does not provide a general response to the Grounding Question. A general response is needed, however, since according to the epistemic conception of progress there are truths that ground theoretical progress - truths that can be specified and known by scientists-whenever there is theoretical progress in science. ${ }^{13}$

Examination of the Grounding Question in connection with some intuitive cases of theoretical progress will reveal the limits of the epistemic conception. ${ }^{14}$ According to Bird, science can progress with approximately true theoretical claims (say, ' $P$ ') as scientists learn new truths of the form ' $P$ is approximately true', thereby accumulating theoretical knowledge. This requires that in all cases of progress scientists can learn truths - truths that constitute the progress made - that can be thus stated on the basis of theories or propositions that are strictly speaking false. This requirement, I will now argue, is implausible. There are cases, first of all, in which scientists for epistemic reasons cannot obtain any clear sense how a theory 'approximates' truth, and therefore they cannot obtain knowledge of this sort (Sect. 3.3). Furthermore, there can also

\footnotetext{
13 Bird (2007) vacillates between different readings of the epistemic conception. Sometimes he seems to equate progress with actual accumulation of knowledge (cf. quote at the beginning), other times with the mere possibility of accumulation subject to 'appropriate epistemic conditions being met' (74). It seems that the latter, weaker reading is the intended one. And the stronger reading seems too strong, indeed; intuitively speaking theoretical progress should not be negated, for example, if scientists chose not to believe their theories regarding the unobservable for some contingent (e.g. sociological) reasons. Bird says little of the 'appropriate epistemic conditions', however.

${ }^{14}$ No doubt that the Grounding Question can be answered in connection with many epistemologically well-founded propositions or theories concerning unobservable matters. The ellipse example is a case in point, of course.
} 
be cases of theoretical progress where talk of 'approximation' seems inappropriate altogether, rendering Bird's move inapplicable (Sect. 3.4). ${ }^{15}$

\subsection{Approaching the truth without accumulation of knowledge}

We can think of various scenarios where theoretical knowledge cannot accumulate along with theoretical progress as per the epistemic conception. Consider the following, for one. Assume we have a theory $T$ which is 'approximately true' (in some contextually specified sense), and a successor theory $T^{\prime}$ that is (by the same measure) even closer to the truth. Assume that the move from $T$ to $T^{\prime}$ is also suitably supported by evidence. Intuitively, this is theoretical progress. This is captured by $T P$ : the better supported theory 'latches better onto reality' in the contextually specified sense of approximate truth.

But the move from $T$ to $T^{\prime}$ need not increase our scientific knowledge so as to capture the theoretical progress being made. The reason is simple: it may be that science, although able to justify $T^{\prime}$ as our best theory, is unable to say how $T^{\prime}$ approximates the truth. It may well be that only from the vantage point of $T^{\prime}$ 's successor, $T^{\prime \prime}$, we can get a handle on how $T^{\prime}$ approximates $T^{\prime \prime}$ (and hence approaches the truth, on the assumption that $T^{\prime \prime}$ is closer to truth than $T^{\prime}$ and its predecessor), even though the move to $T^{\prime}$ from its predecessor was progressive. In more general and abstract terms, we can put the point as follows: it may be that scientific method is reliable in producing better theories in the relevant sense of approximate truth, without providing scientists even roughly synchronous ability to know the relevant truths about the approximation. Without a clear sense of the way in which their theory approximates the truth, scientists are unable to form any clear belief-never mind true belief that could qualify as knowledge-about the theory being approximately true. Since the theory is not fully true, it cannot in and of itself be an object of knowledge. And a truth of the form 'the theory is approximately true' (in the relevant sense of 'approximately true') may not be epistemically accessible to scientists at that time. So, theoretical progress could be based on $T^{\prime}$ 's factual approach to truth (in conjunction with suitable evidential support), even if $T^{\prime}$ 's approximate truth cannot for epistemic reasons support accumulation of knowledge of the sort that captures this progress. ${ }^{16}$

Are we indulging in mere abstract speculation about an unrealistic conceptual possibility? Certainly not. There are momentous precedents of this possibility in actual science. As Barrett (2008) carefully explains, Newtonian gravitation furnishes an excellent illustration of this kind of progress in fundamental physics. While the descriptions of the world afforded by Newtonian gravity (NG) and the general theory of relativity (GTR) are radically at odds with one another, there are also now wellunderstood descriptive similarities between the two theories that provide a contextual,

\footnotetext{
15 Niiniluoto (2014) objects to Bird's move, briefly but incisively, very much in the spirit the objections developed in more detail below.

16 Could scientists perhaps be in a position to know that a theory is approximately true, even in the absence of any knowledge of how it is approximately true? I am not sure. But it doesn't matter, since such accumulation of knowledge, even if possible, does not comprise the intuitive sense of theoretical progress at stake.
} 
local sense in which NG can be construed as 'approximating' GTR. But even if these descriptive similarities furnish a sense in which NG as a matter of fact is 'approximately true', it is not a sense that Newton could have grasped. It is only now, from the perspective of GTR, that we can get a handle on this sense of approximation.

The dissimilarities between NG and GTR are striking and routinely used as a reminder that a 'radically false' theory can be highly empirically adequate. According to NG a body of mass $m$ is accelerated towards a body of mass $M$ due to a gravitational force that is inversely proportional to their distance $r$ squared:

$$
G \frac{m M}{r^{2}}
$$

According to GTR, by contrast, the two bodies experience no force nor acceleration, but rather follow a locally straight trajectory (geodesic) in curved spacetime. Only if there is some non-gravitational force will the bodies divert from this natural, 'unforced' state of motion.

Despite this radical dissimilarity in their theoretical descriptions and fundamental ontology, NG and GTR also share significant (extra-empirical) similarities. As Barrett (2008) explains_-following Malament's work on the topic_-our understanding of these similarities is in large part due to the post-GTR development of a geometrized version of Newtonian gravitation (GNG), which is empirically equivalent to NG but absorbs all the information about gravitational forces into information about spacetime curvature so that gravitation is rendered a manifestation of spacetime curvature as in GTR. GNG furnishes us with a precise sense of the inter-theoretic correspondence between NG and GTR, allowing for a detailed comparison of the respective gravitational field equations, bringing out, for example, the way in which for empty space the gravitational field equation of GTR is extremely closely related to the field equation of NG (Ibid., 219). Similarly, GNG furnishes us with a precise and theoretically rich sense in which NG is a non-relativistic limit of GTR: the field equation of NG, in its geometrized form, is recovered at the limit of Einstein's field equations as relativistic effects vanish. Even the Euclidean character of Newtonian space is determined by a well-defined classical limit of GTR. ${ }^{17}$

Armed with a precise understanding of the correspondence between NG and GTR it makes sense to refer to the features of NG that are preserved in GTR. Reflecting now on Newton's epistemic situation, I fully agree with Barrett's verdict that for a Newtonian realist who considers the theory to be 'approximately true',

the expected preservation [in future theories] of each of these features is presumably part of what Newton should have wanted to mean in claiming that his account of gravitation was at least approximately true. Of course, Newton could

\footnotetext{
17 See Malament (1986a, b) for details. 'Relativistic effects' are captured in terms of the light cone geometry at each spacetime point in a solution of Einstein's field equation as the upper bound of particle speed goes up. The Newtonian limit is a fully 'flattened' light cone-as the upper bound for particle speed goes to infinity-that is tangent to a hypersurface that represents a three-dimensional space at a time. Throughout the limiting process spacetime structure must conform with the dynamic constraints of GTR. As Malament (1986a) puts it: 'The limiting process which effects the transition from general relativity to Newtonian gravitational theory "squeezes out" all spatial curvature!' (406).
} 
not have meant anything so precise without knowing what it would take to translate between descriptions in NG and descriptions in subsequent theories, and he did not know this. (220, my emphasis)

But while Newton only had quite limited, partial knowledge of the way in which his theory latches onto reality (as described by GTR), from our later vantage point we can provide a rich and precise account of the empirical accuracy of Newton's theory in terms of some of its key features 'approximating' GTR (This is how work on the foundations of physics naturally gets involved in the realism debate!).

This example from the history of science shows concretely how Bird's move can fail with respect to truths of the form ' $T$ ' is approximately true'. But is there some other kind of pertinent increase in theoretical knowledge that necessarily accompanies the progressive move from $T$ to $T^{\prime}$ ? An advocate of the epistemic conception of progress might argue along the following lines that there is. In adopting $T^{\prime}$, scientists have at least learned some specific truths pertaining to $T$ and its failures. Scientists have learned, in particular, how some specific aspects of $T$ are in error by virtue of adopting $T^{\prime}$ that overcomes (some of) the empirical limitations of $T$ by virtue of appropriately differing from $T$. And this is a guaranteed form of accumulation of theoretical knowledge in the shift from $T$ to $T^{\prime}$, even if scientists are not in a position to say how $T^{\prime}$ is approximately true.

Although it is important to recognise accumulation in this kind of 'Popperian' theoretical knowledge, it is not enough to salvage the epistemic conception of progress. Even if this type of knowledge accumulation is guaranteed in theory change, it does not seem right to think that such knowledge would ground all the theoretical progress associated with the move from $T$ to $T^{\prime}$. For intuitively it seems that theoretical progress here at least in part has to do with what $T^{\prime}$ 'gets right' about the world-how $T^{\prime}$ represents an improvement over $T$ relative to the aim of providing a good representation of unobservable reality_not with what is known with the help of $T^{\prime}$ to be definitely wrong about $T$. The fact that we can associate (some of) the empirical limitations of $T$ clearly with some of its mistaken theoretical assumptions does not seem to be all that is progressive about adopting $T^{\prime}$ in the face of the evidence. Rather, some of the theoretical progress made with $T^{\prime}$ intuitively has to do with the way in which $T^{\prime}$ latches better onto reality - as per $T P$ - in a way that is responsible for its empirical success that provides empirical support for it over $T$. And to have knowledge of this would require knowledge of how the theory latches onto reality, how it is approximately true. In the case of Newton, for instance, part of the theoretical progress of NG has to do with the way in which the theory gives basically the correct field equation for relativistic empty space, for example. ${ }^{18}$

\footnotetext{
18 Harker (2013) argues that even if we cannot know whether a theory $T^{\prime}$ is approximately true, we can know-and in order to be realists we must know - the approximate truth of those new elements of $T^{\prime}$ the introduction of which is responsible for the empirical progress that $T^{\prime}$ makes in relation to $T$. I fail to see how knowledge of this sort is conceptually required by realism (cf. Sect. 5 below), and I fail to see how Harker's view can accommodate revolutionary wholesale shifts in theories, such as the one exemplified by the shift from Aristotelean mechanics to Newton's theory. (There's a considerable overlap between some other aspects of Harker's position and mine, though.)
} 
We have found a decisive reason to deem the epistemic conception inadequate: scientists can make theoretical progress without accumulating knowledge of how their theories are better latching onto reality. This, I will next explain, is further exacerbated by the fact that theories can latch onto reality (so as to constitute theoretical progress) in ways that are not amenable to Bird's move, regardless of the epistemic limitations discussed above.

\subsection{Progress with radically false theories}

It is plausible that some theories can be so far off the mark, so radically false, that in no reasonable sense can we think of them as 'approximating' the truth. Such radically false theories could nevertheless exhibit theoretical progress quite unproblematically, if an empirically better supported theory makes better contact with the unobservable reality (in a way that isn't accidental). This is readily captured by $T P$ which takes progress to be a matter of a particular kind of improvement that can be exhibited by radically false and approximately true theories alike. The epistemic conception, on the other hand, again faces the Grounding Question: what known truths ground this progress? If the theories in question cannot be said to be approximately true to begin with, then one cannot make Bird's move and point to a knowable true proposition ' $T$ approximates the truth thus-and-so' to capture the way these theories latch onto reality and the improvement therein. Perhaps Newtonian gravitation as a matter of fact is best regarded as a radically false theory that makes theoretical progress. Bird's move rings hollow to those disillusioned with the extant attempts to sensibly speak of theories such as Newton's as 'approximately true'.

One might argue that a theory can conceivably be radically false in this way only if we are operating with an overly simplistic, naive, or restrictive notion of approximate truth. Arguably a sophisticated and versatile account of verisimilitude-such as the accounts developed by Niiniluoto, Kuipers, and others-would allow us to speak of any theory's verisimilitude to capture its 'distance from the truth', even in the case of (intuitively) radically false theories. ${ }^{19}$ It might be thought that this provides the required basis for strictly true propositions that can function as objects of knowledge: e.g. ' $T$ has such-and-such degree of verisimilitude, while $T^{\prime}$ has thus-and-so.' More generally, in as far as radically false theories can be construed as latching onto unobservable reality at all, there will have to be corresponding true propositions that capture this. It might be thought that the epistemic conception can capitalise on these truths as the relevant objects of knowledge.

But this is not right: these truths about formal measures of verisimilitude or the various ways in which theories latch onto unobservable reality do not allow us to defend the epistemic conception by an extension of Bird's move. This move derives its initial plausibility from relatively straightforward cases like 'planets travel in ellipses' in which an unproblematic, rough-and-ready conception of approximation naturally accompanies the theory in question. Contrast this with the rarefied sense in which we can now understand, through GTR, Newton's theory latching onto reality. It is

${ }^{19}$ See e.g. Niiniluoto (2002), Kuipers (2000). Cevolani and Tambolo (2013) and Niiniluoto (2014) explicitly discuss, in response to Bird, the importance of the expected verisimilitude measure. 
totally implausible that a truth about such an opaque sense of verisimilitude-about such a qualified sense of 'latching onto reality' - could be epistemically accessible to scientists who make theoretical progress by virtue of propounding a better (even if still radically false) theory in the face of the evidence. Therefore, even if there are truths about increasing verisimilitude in some well-founded formal sense, or truths about how theories latch onto reality in general, theoretical progress cannot be equated with accumulation of knowledge of such truths.

It is pertinent at this point to flag the importance of clarifying the sense of improvement associated with the notion of theoretical progress. Bird (2007) wants to exploit the key virtue of the verisimilitude account of progress by showing how to move from increasing verisimilitude to the (possibility of) accumulation of knowledge. (76-78) The extant verisimilitude accounts face a significant challenge, however: how to justify the equation of progress with increasing verisimilitude? The problem is that measures of verisimilitude and judgements of increase/decrease in verisimilitude turn out to be language dependent (e.g. Miller 1975). ${ }^{20}$ Assume for the sake of the argument that relative to a particular language our preferred formal account yields a particular degree of verisimilitude for the old theory, and another for the new one. Assume the verisimilitude goes up. Why exactly should this be regarded as constitutive of theoretical progress?

I will not attempt here to assess the responses made on behalf of the verisimilitude account. (e.g. Mormann 1988; Niiniluoto 2002) I just want to point out that by exploiting the verisimilitude account Bird inherits this challenge: it is not clear why knowledge of increasing verisimilitude (relative to a given language) - even if scientists could attain such knowledge-would automatically constitute the progress made through these theories. Answering this challenge requires spelling out a sense in which the given language-relative increase in verisimilitude represents an objective improvement in theories. In as far as a formal account of verisimilitude can be used to precisify my vague and metaphorical notion of theories 'latching onto reality', I obviously face the challenge likewise. I will not consider this exact issue here. Instead, I will now address the (more general) question I flagged above, regarding the sense of improvement we associate with theoretical progress: what does it mean for a theory to latch better onto reality than another?

\section{Latching (better) onto reality}

I have been arguing that one important dimension of theoretical progress has to do with how well theories represent the unobservable world: science makes theoretical progress if a better confirmed theory latches better onto reality than its predecessor. It is high time to elucidate this hitherto worryingly metaphorical and nebulous notion.

Capturing the way in which theories depict reality, better or worse but practically never perfectly, is of course one of the deepest issues in the philosophy of science. Here I can't even begin to do justice to the enormous complexities involved in the various

\footnotetext{
20 Furthermore, a (prima facie) progressive theory change might also be associated with a linguistic revision, as in the case of the shift from Newton to Einstein.
} 
attempts to capture in precise terms the way in which theories latch onto reality. Luckily, I don't have to. The trick is to acknowledge that for my present purposes it is admissible to operate at a relatively high level of abstraction, just focusing on TP and what it requires of 'latching' (leaving it open how exactly this notion could be further precisified in different formal frameworks, for example). In particular, what matters is that we can pin down an objective sense in which one theory can be construed as latching better onto unobservable reality than another, so as to give objective meaning to theoretical progress as expressed by $T P$. I will proceed by presenting a broad twofold definition that guarantees objectivity and gives $T P$ substantive enough content.

Let's begin by stipulating a sense in which we can talk of a theory $T$ latching onto unobservable reality.

$T$ LATCHES ONTO UNOBSERVABLE REALITY if and only if $T$ 's degree of empirical adequacy is accounted for by $T$ providing a veridical representation of some aspects of unobservable reality.

Given this definition, we can then further stipulate that:

$T^{\prime}$ LATCHES BETTER ONTO UNOBSERVABLE REALITY than $T$ if and only if $T^{\prime}$ is more empirically adequate than $T$, and the boost in empirical adequacy is accounted for by a difference in the respective provisions of veridical representations.

Let's unpack the definition of LATCHING a bit. We have a theory $T$ that represents the unobservable reality being, perhaps amongst (many) other things, thus-and-so. $T$ has a certain degree of empirical adequacy. If $T$ 's empirical adequacy is accounted for by the reality really being thus-and-so, then we say that $T$ latches onto reality, period. Never mind if we are not in a position to provide an account of T's empirical adequacy in these terms. Never mind if the scientists upholding $T$ cannot see how the theory thus latches onto reality; they often cannot, lacking a true theory to compare $T$ with. Never mind what 'proportion' or 'part' of $T$ 's representational content is involved in accounting for its empirical adequacy; sometimes $T$ 'says' many irrelevant things about the reality behind a phenomenon (as in the case of some of the 19th c. ether theories). Never mind that LATCHING does not aim to capture any kind of 'overall correspondence' between a theoretical representation and reality, but rather is relative to $T$ 's empirical adequacy. Never mind that there may not be any obvious, straightforward connection between how $T$ represents the unobservable world, on the one hand, and what accounts for its empirical adequacy, on the other. ${ }^{21}$

With this definition in hand, LATCHING BETTER is then just a matter of latching onto reality and being more empirically adequate. Since the respective degrees of empirical adequacy of $T$ and $T^{\prime}$ is accounted for by them providing a veridical representation

\footnotetext{
21 A toy example: One is theorising about an unobservable (neural, say) network with 11 nodes. Assume that a certain feature of the network only depends on the number of nodes being a prime number between 1020 , and that this feature is revealed through a phenomenon the prediction of which depends on latching onto the primality of nodes. With respect to this phenomenon $T_{19}$ nodes latches onto reality, while $T_{12}$ nodes does not. This is not going to be at all obvious to anyone ignorant of the significance of primality. For example, one might well take the predictive success of $T_{19}$ nodes over $T_{12}$ nodes as an indicator of $T_{19}$ nodes getting the number of nodes almost right.
} 
of some aspects of unobservable reality, the increase in empirical adequacy is also accounted for by differences in the respective accounts of empirical adequacy. The boost in empirical adequacy can be due to various kinds of theoretical improvements. For example, it can be due to $T^{\prime}$ providing a veridical representation of further pertinent aspects of reality (e.g. additional mechanistic detail). Or, it can be due to $T^{\prime}$ providing a more fine-grained representation of the same aspects of reality (e.g. by incorporating additional variables to quantify fluctuations at a shorter time-scale). Whatever the details, at the bottom LATCHING BETTER is just a matter of $T^{\prime}$ being more empirically adequate by virtue of latching onto reality in a way that also accounts for the boost in empirical adequacy.

LATCHING (BETTER) ONTO REALITY is a notion intimately connected to the explanationist realist agenda. In particular, the realist's basic motivation, articulated in the 'no-miracles' argument, turns on the intuition that predictive success (suitably construed) would be extremely unlikely, or difficult to explain, unless theories were latching onto unobservable reality in ways that account for the success. The nomiracles intuition, and the different arguments that capitalize on it, say little about what kinds of theory/world relations satisfy the explanatory request at stake. With this in mind, the notion of LATCHING (BETTER) ONTO REALITY is defined in broad terms, to capture all (and only) the gist of the no-miracles intuition. In particular, it is meant to be compatible with various forms of scientific realism that are all in the business of accounting (in realist terms) for the empirical success of false past theories from our current perspective. Different realists can offer different, more precise definitions that take a stand on exactly what kind of representational adequacy can account for a theory's degree of empirical adequacy (in a way that satisfies the realist intuitions and doesn't give the game away to the anti-realist). The advocates of structural realism, for example, claim that the empirical success of past theories can be accounted for in terms of these theories providing a veridical representation of critical structural aspects of reality. ${ }^{22}$ A related but subtly different position claims that the empirical success of past theories is (sometimes) best accounted for in terms of these theories providing a veridical representation of critical less specific properties. (Saatsi 2005) Both realist positions are committed to the claim that the degree of empirical adequacy enjoyed by Fresnel's optical ether theory, for example, can be accounted for in terms of Fresnel's theorising latching onto reality. Similarly, the boost in empirical adequacy achieved by classical electrodynamics - itself a false theory as a classical (non-quantum) theorycan arguably be accounted for in terms of the theory latching better onto reality. These different realist positions all claim that there is an unambiguous sense of theoretical progress from Fresnel to Maxwell to Feynman; the disagreement is mainly about how to best capture the specific sense in which these theories are progressively latching onto reality.

This progress is captured by $T P$ regardless of one's specific realist preferences. ${ }^{23}$ According to the realists Fresnel made theoretical progress, for example, since his

\footnotetext{
22 There are various differences amongst the structural realists. For a review, see Frigg and Votsis (2011).

$23 \mathrm{TP}$ is also meant to be compatible with pluralism regarding different philosophical, meta-scientific frameworks that can be used to capture 'latching onto' in more specific terms. These include, for example,
} 
theoretical assumptions latched onto reality better than those of his predecessors. Fresnel's progress does not boil down to Fresnel knowing —or having the possibility of knowing 'had suitable epistemic conditions been met' (cf. Bird 2007, p. 76) truths about what light really is like and why it really behaves the way it does. Fresnel made theoretical progress even if Laudan (1981) is right in claiming that there is no sense in which Fresnel's theory can be considered 'approximately true', and even if Fresnel was in no position to formulate-never mind know-relevant truths of the sort 'approximately, light is such-and-such waving of the ether'.

There's obviously much to be said about how the fact that a theory provides a veridical representation of some features of unobservable reality accounts for the theory's empirical adequacy. The realist notion that only some 'parts' or 'aspects' of a theory can be selectively responsible for (and account for) its predictive success is delicate, but it is commonly accepted that the realist has to appeal to such a notion in order to square the impressive predictive success of science with various kinds of falsehoods involved in the past and present science. I have nothing further to add here to the extant attempts to get to grips with this notion, except to note again that scientists working on inter-theory relations could often be viewed as directly contributing to these attempts; the task isn't just a conceptual one for philosophers to sort out-it is part and parcel of theoretical science itself! Although there is further work to be done here, I share the realist optimism that sense can be made of the idea that, for example, only a relatively small subset of Augustine Fresnel's theoretical assumptions regarding the optical ether enter into a realist explanation of Fresnel's predictive successes.

\section{Realism and progress}

There is an intimate conceptual affinity between scientific realism and theoretical progress, and the foregoing analysis of the latter has significant repercussions regarding our understanding of realism.

It is natural to understand scientific realism as being committed, at the minimum, to the claim that science as a matter of fact makes theoretical progress. ${ }^{24}$ This is also how Bird (2007) understands realism, but he of course frames it in epistemic terms, in terms of knowledge of the unobservable.

[Realism can be taken to claim] that there has been progress in regard of our knowledge of theoretical propositions concerning the unobservable. ${ }^{25}$ (79)

Most commentators have likewise associated realism with growing scientific knowledge. For example, in a recent review of scientific realism, Chakravartty (2011) expounds:

\footnotetext{
Footnote 23 continued

(i) the similarity approach (e.g. Giere 1988; Teller 2001); (ii) the partial isomorphism approach (e.g. Da Costa and French 2003); (iii) the mathematico-logical structure approach (Worrall 2007).

24 This is part of the motivation for defining 'theoretical progress' as extra-empirical, extra-instrumental, extra-pragmatic (cf. Sect. 1).

25 Bird also considers the broader realist thesis that 'science has always progressed', but as he notes, this is compatible with anti-realist positions like constructive empiricism.
} 
Most commonly [realism] is described in terms of the epistemic achievements constituted by scientific theories ...What all of approaches [to defining realism] have in common is a commitment to the idea that our best theories have a certain epistemic status: they yield knowledge of aspects of the world, including unobservable aspects. (my emphasis)

Conceptualising realism thus in terms of accumulation of knowledge is extremely widespread. We could call it the standard conception. Its popularity is not surprising; admittedly it is natural and intuitive to equate realism with the idea that scientists constantly learn new facts about the unobservable world. But natural though this equation is, it is again too simple. For it is also natural to think of realism just in terms of theoretical progress of science, and we have seen that there is a dimension of theoretical progress that has to do with the way in which theories latch onto reality that cannot always be captured in terms of accumulation of scientific knowledge. Corresponding to this further dimension of progress there is an epistemologically weaker realist claim: theories actually latch onto reality better and better so as to drive our theories' increasing empirical adequacy.

This conception of realism is more minimal than the standard conception. ${ }^{26}$ It is not meant to replace the standard conception, but rather to extend or complement it. The complement matters, because the standard conception fails to fully capture the theoretical progress (conceivably) made by some of the most fundamental scientific theories and the appropriate epistemic commitments towards those theories. ${ }^{27}$

Newtonian gravity is again a case in point. What kind of realist commitment towards Newtonian mechanics would not have been countered by the subsequent scientific developments? According to the standard conception, the realist is committed solely to the idea that Newton's theory yields knowledge of some unobservable aspects of reality. But what kind of knowledge of the unobservable could Newton and his contemporaries possibly have had? All the ontological presuppositions of Newton's theoretical framework are simply false: there is no gravitational force, no spacetime 'background' relative to which things move, no absolute simultaneity. And the New-

\footnotetext{
26 More precisely, it is a more minimal conception of theory-realism. There are more minimal forms of entity realism, for example, which lie outside the scope of this paper. Some also regard as realism some declarations of knowledge of certain systematic counterfactual truths regarding observable matters. For example, Northcott (2013) discusses scientific progress in light of such conception of 'causal verisimilitude', and Harper (2011) discusses in great detail the progress made by Newton by these lights. Woodward (2003) calls such position 'instrumental realism'. It is a matter of debate to what extent those declarations and the associated accumulation of knowledge transcend constructive empiricism in any substantial way. (Cf. Huggett's (2013) review of Harper.)

27 Cf. Barrett (2008):

'If the standard collapse formulation of quantum mechanics and relativity are almost certainly false taken together and if, since we do not know how to fix them, we do not know the sense in which they can be taken to be approximately true, then in what sense do our current best physical theories provide physical knowledge?' (215)
}

Barrett's response is to capture our current commitments and knowledge in terms of imprecise 'descriptive nesting relations' that can only be made precise from a later vantage point. Barrett's view is closely related to my sense of theories latching onto reality. See also Rohrlich and Hardin (1983). 
tonians could not have known the subtle sense in which we can now grasp Newton's theory as having latched onto reality as described by Einstein's general relativity.

A case in point, indeed, but a case against realism, cries the anti-realist! Not only is the key theoretical term 'gravitational force' seemingly non-referring, but in as far as we may occupy a similar epistemic predicament regarding our current fundamental theories, arguably even structural realism is bankrupt since 'we will not know what [structural] aspects of current theories are the ones we can safely regard as accurately describing features of the natural world.' (Stanford 2003, p. 570, my emphasis)

The anti-realist must concede that some new truths about the unobservable world were correctly believed by (or epistemically accessible to) the Newtonians. ${ }^{28}$ But she may insist that the knowledge grounded in these truths is negligible in the face of the overall dialectic of the realism debate. In as far as the realist is driven by some kind of no-miracles intuition regarding novel predictive successes and the theory's overall empirical adequacy, what matters is that the realist commitments are geared towards accounting for these successes. And it is not at all clear - the anti-realist presses - that Newton's actual and potential knowledge gains can do that. For example, the fact that Newton learned that tidal and planetary phenomena are of the same, gravitational origin-without knowing what that origin actually is - can hardly account for the overall predictive accuracy of the theory or for Newton's novel prediction that Earth is such-and-such an oblate spheroid. And arguably the rich modal knowledge gained by Newton does little beyond summarising (one aspect of) the 'miracle' of the nomiracles intuition: that the theory is empirically very accurate with respect to a huge range of actual and possible configurations of massive bodies.

The anti-realist reaction is a step too far, however. Realist commitments need not be concerned with 'first-order' scientific knowledge about this or that unobservable feature of the natural world, as suggested by the standard conception of realism. As a global attitude towards science, realism can merely be committed to our fundamental theories making theoretical progress by virtue of suitably latching onto reality. (See Saatsi 2016.) And arguably Newton's theory did latch onto unobservable reality in complicated and highly non-trivial ways that account for the theory's empirical accuracy, but in ways that we can only appreciate and study from later vantage points, by studying its inter-theoretic relationship to general relativity, for example. (Malament 1986a, b, 2012) Our current vantage point need not be privileged in any way, either as 'a final theory', or as a theory that we know to be approximately true in some specific sense. But come what may, if science actually makes theoretical progress in a way a sensible realist believes it does, then we will always be able to account for past theories' increasing empirical adequacy, with hindsight as it were, in these terms.

Such minimal realism is still an epistemological attitude, of course, but its key knowledge claim about the world is somewhat indirect: what we know of the unobservable reality, says the realist, is that our best theories are latching onto it - in the sense explicated above (Sect. 4). We can further precisify this global realist attitude locally,

\footnotetext{
28 Newton gained knowledge of the fact that projectile and tidal phenomena are ultimately of the same origin, for example. More generally, Newton gained knowledge of remarkable systems of fine-grained counterfactual truths that count as causal truths in some prominent accounts of causation. (Harper 2011; Woodward 2003). See also footnote 26.
} 
with particular exemplars, perhaps also taking into account one's preferences for a specific meta-scientific framework to analyse theory-world relationships. This belief concerns squarely the unobservable, and it transcends mere instrumentalist and empiricist commitments regarding the observable, typically cashed out in terms of increasing empirical adequacy and the representational faithfulness of theories' empirical substructures. (See Saatsi 2015 for related discussion.) Along with the realist position comes also a commitment to try to figure out the ways in which theories are thus latching onto reality, so that future theorising can latch onto reality even better. For the realist scientists' employment and research into various kinds of 'correspondence rules' is not just a matter of pragmatics! ${ }^{29}$

Delineating such a minimal realist position is one thing, defending it is another. I will not attempt to construct or assess a positive case for realism here. Rather, I have been concerned with the ground work: getting clear on what realism could be. This ground work is significant in clarifying some of the misconceptions in the current literature. For example, at the heart of Stanford's (2003) forceful critique of realism is the worry that contemporary scientists cannot trust their current theories to give them knowledge-this is Stanford's trust argument. As far as I can judge, the main advocates of structural realism, for instance, have not committed (and need not commit) themselves to the kind of epistemic trust that Stanford takes to be constitutive of realism. The right way to understand structural realism, I propose, is as a form of minimal realism.

\section{Further dimensions of theoretical progress?}

Progress, in general, is a result-oriented, evaluative concept that applies to a positive achievement. This concept is so open-ended that it is reasonable to expect scientific progress to frustrate attempts to fully capture it in universal terms. The epistemic conception offers such a universal, one-size-fits-all account of scientific progress. ${ }^{30}$ I have argued that there is a dimension of theoretical progress that doesn't fit this conception. Accumulation of knowledge is one important dimension of scientific progress, for sure. The way in which theories latch better and better onto unobservable reality is another. Are there yet further dimensions?

I think so. My discussion of TP has focused on the way in which 'latching onto reality' can be cashed out in terms of a theory's representational veridicality accounting for its empirical accuracy. This was inspired by the fact that all (theory) realists are in one way or another committed to accounting for a theory's predictive accuracy. But there are other aspects of 'empirical success' a realist could also be committed to. For example, one might focus instead on improved theoretical understanding as (a dimension of) theoretical progress. ${ }^{31}$

\footnotetext{
29 See Hartmann (2002) for different kinds of correspondence principles operating in science, and Rohrlich and Hardin (1983) for application to realism.

30 Northcott (2013) offers a different one-size-fits-all account, in terms of increasing causal verisimilitude.

31 See also Rowbottom (2015) for related remarks.
} 
Shifting the focus from prediction to understanding and explanation raises difficult issues that I leave gladly for later work. As a precursory note, however, I wish to briefly comment on Bird's attempt to incorporate understanding into the epistemic conception of progress.

[W]hile the importance of understanding is clear, that does not contrast with the aim of knowledge, because all (genuine as opposed to apparent) understanding is also knowledge. To understand why something occurred is to know what causes, processes, or laws brought it about. (84)

Conceptualising scientific understanding in these terms is widespread. ${ }^{32}$ But there's room to resist this equation of increased understanding with accumulating knowledge (of laws, causation, processes); increase in understanding can plausibly transcend accumulation of knowledge in interesting ways. Consider explanatory idealisations, for example. It is a striking fact that by incorporating idealisations science can sometimes improve our understanding despite taking us away from the truth (e.g. Elgin 2004, 2007). Bird's move, by a natural extension, would admit that these idealisations involve felicitous approximations, but maintain that theoretical progress qua increased understanding is underwritten by knowable truths of the form 'approximately $P$ '. But it is not at all clear whether this move can be sustained. The problem is that scientists have been seemingly able to obtain progress-inducing understanding from idealisations without having knowledge of the sense of 'approximation' at play.

Take, for instance, our understanding of the phenomena captured by the ideal gas law. A standard textbook explanation of the ideal gas law conveys an important part of that understanding by representing gas molecules as point particles that never collide with one another and only bump the container walls. This is quite a radical idealisation, since intermolecular collisions are actually rather frequent. Did the ideal gas model convey understanding only when scientists grasped the way in which the model relates to reality and why it furnishes an explanatory derivation of the ideal gas law despite its radically idealised assumptions? A contestable claim, to say the least.

Some prominent cases of explanatory idealisation involve more radical falsehoods that are even less amenable to Bird's move (see e.g. Batterman 2002; Bokulich 2008; Rice 2015). Although the explanatory contribution of these idealisations (involving e.g. asymptotic limits) is a matter considerable debate, some clearly take them to support Elgin's claim about the non-factivity of understanding, according to which the full cognitive contribution to theoretical understanding cannot be captured by truths alone (2004, 2007). ${ }^{33}$ On the face of it, the epistemic conception of progress appears too simple in this dimension as well.

\footnotetext{
32 E.g. Lipton (2004, p. 30): 'Understanding is not some sort of super-knowledge, but simply more knowledge: knowledge of causes.'

33 Elgin herself (effectively) denies Bird's move in relation to Rawls's theory of justice, for example.
}

$[\mathrm{N}]$ ot all felicitous falsehoods are approximations. Idealizations may be far from the truth, without thereby being epistemically inadequate. Political agents are not mutually disinterested. They are not nearly mutually disinterested. Nor is it the case that most political agents are mutually disinterested. There is no way I can see to construe Rawls's model as approximately true. Nevertheless, for 
Acknowledgements A version of this paper was presented at York University workshop on scientific realism, and at colloquia at UCSD and UC Irvine. I would like to thank the audiences. I would also like to thank two anonymous reviewers for their helpful comments. The research has been partly funded by AHRC Realism and the Quantum project.

Open Access This article is distributed under the terms of the Creative Commons Attribution 4.0 International License (http://creativecommons.org/licenses/by/4.0/), which permits unrestricted use, distribution, and reproduction in any medium, provided you give appropriate credit to the original author(s) and the source, provide a link to the Creative Commons license, and indicate if changes were made.

\section{References}

Barrett, J. (2008). Approximate truth and descriptive nesting. Erkenntnis, 68(2), 213-224.

Batterman, R. W. (2002). Asymptotics and the role of minimal models. British Journal for the Philosophy of Science, 53(1), 21-38.

Bird, A. (2007). What is scientific progress? Noûs, 41, 64-89.

Bird, A. (2008). Scientific progress as accumulation of knowledge: A reply to rowbottom. Studies In History and Philosophy of Science Part A, 39(2), 279-281.

Bokulich, A. (2008). Reexamining the quantum-classical relation: Beyond reductionism and pluralism. Cambridge: Cambridge University Press.

Cevolani, G., \& Tambolo, L. (2013). Progress as approximation to the truth: A defence of the verisimilitudinarian approach. Erkenntnis, 78(4), 921-935.

Chakravartty, A. (2011). Scientific realism. Stanford Encyclopedia of Philosophy.

Da Costa, N., \& French, S. (2003). Science and partial truth: A unitary approach to models and scientific reasoning. Oxford: Oxford University Press.

Douglas, H. (2014). Pure science and the problem of progress. Studies in History and Philosophy of Science, 46, 55-63.

Elgin, C. (2004). True enough. Philosophical Issues, 14, 113-131.

Elgin, C. (2007). Understanding and the facts. Philosophical Studies, 132(1), 33-42.

Frigg, R., \& Votsis, I. (2011). Everything you always wanted to know about structural realism but were afraid to ask. European Journal for Philosophy of Science, 1(2), 227-276.

Giere, R. (1988). Explaining science: A cognitive approach. Chicago: University of Chicago Press.

Harker, D. (2013). How to split a theory: Defending selective realism and convergence without proximity. The British Journal for the Philosophy of Science, 64(1), 79-106.

Harper, W. (2011). Isaac Newton's scientific method: Turning data into evidence about gravity and cosmology. Oxford: Oxford University Press.

Hartmann, S. (2002). Essay review: On correspondence. Studies in History and Philosophy of Modern Physics, 33, 79-94.

Huggett, N. (2013). On newton's method. Metascience, 22(2), 215-246.

Jones, N. (2013). Don't blame the idealizations. Journal for General Philosophy of Science, 44, 85-100.

Kuipers, T. (2000). From instrumentalism to constructive realism. On some relations between confirmation, empirical progress, and truth approximation. Dordrecht: Kluwer.

Laudan, L. (1981). A confutation of convergent realism. Philosophy of Science, 48, 19-49.

Leplin, J. (1984). Scientific realism. Los Angeles: University of California Press.

Lipton, P. (2004). Inference to the best explanation (2nd ed.). London: Routledge.

Malament, D. (1986a). Gravity and spatial geometry. In R. Barcan Marcus, G. J. W. Dorn \& P. Weingartner (Eds.), Logic, methodology and philosophy of science VII (pp. 405-411). Amsterdam: Elsevier.

Footnote 33 continued

Rawls's purposes, the characterization of political agents as mutually disinterested is felicitous if the features it highlights are constitutive of fair terms of cooperation underlying the basic structure of a democratic regime. There is no reason to think that in general the closer it is to the truth, the more felicitous a falsehood. (2004, p. 125). 
Malament, D. (1986b). Newtonian gravity, limits, and the geometry of space. In R. Colodny (Ed.), From quarks to quasars: Philosophical problems of modern physics (pp. 181-201). Pittsburgh: University of Pittsburgh Press.

Malament, D. B. (2012). Topics in the foundations of general relativity and Newtonian gravitation theory. Chicago: University of Chicago Press.

Miller, D. (1975). The accuracy of predictions. Synthese, 30(1), 159-191.

Mizrahi, M. (2012). Idealizations and scientific understanding. Philosophical Studies, 160, $237-252$.

Mizrahi, M., \& Buckwalter, W. (2014). The role of justification in the ordinary concept of scientific progress. Journal for General Philosophy of Science, 45, 151-166.

Mormann, T. (1988). Are all false theories equally false? A remark on David Miller's problem and geometric conventionalism. The British Journal for the Philosophy of Science, 39(4), 505-519.

Niiniluoto, I. (2002). Critical scientific realism. Oxford: Oxford University Press.

Niiniluoto, I. (2011). Scientific progress. In E. Zalta (Ed.), Stanford Encyclopedia of Philosophy.

Niiniluoto, I. (2014). Scientific progress as increasing verisimilitude. Studies in History and Philosophy of Science, 46, 73-77.

Northcott, R. (2013). Verisimilitude: A causal approach. Synthese, 190(9), 1471-1488.

Rice, C. (2015). Moving beyond causes: Optimality models and scientific explanation. Noûs, 49(3), 589615.

Rohrlich, F., \& Hardin, L. (1983). Established theories. Philosophy of Science, 50(4), 603-617.

Rowbottom, D. (2008). N-rays and the semantic view of scientific progress. Studies In History and Philosophy of Science, 39, 277-278.

Rowbottom, D. P. (2010). What scientific progress is not: Against bird's epistemic view. International Studies in the Philosophy of Science, 24(3), 241-255.

Rowbottom, D. P. (2015). Scientific progress without increasing verisimilitude: In response to Niiniluoto. Studies in History and Philosophy of Science, 51, 100-104.

Saatsi, J. (2005). Reconsidering the Fresnel-Maxwell case study. Studies in History and Philosophy of Science, 36, 509-538.

Saatsi, J. (2015). Historical inductions. Old and new. Synthese. doi:10.1007/s11229-015-0855-5.

Saatsi, J. (2016). Replacing recipe realism. Synthese. doi:10.1007/s11229-015-0962-3.

Stanford, P. K. (2003). Pyrrhic victories for scientific realism. Journal of Philosophy, 100(11), 553-572.

Teller, P. (2001). Twilight of the perfect model model. Erkenntnis, 55(3), 393-415.

van Fraassen, B. (1980). The scientific image. Oxford: Oxford University Press.

van Fraassen, B. C. (2003). On McMullin's appreciation of realism concerning the sciences. Philosophy of Science, 70, 479-492.

Woodward, J. (2003). Experimentation, causal inference, and instrumental realism. In H. Radder (Ed.), The philosophy of scientific experimentation (pp. 87-118). Pittsburgh: University of Pittsburgh Press.

Worrall, J. (2007). Miracles and models: Why reports of the death of structural realism may be exaggerated. In A. O'Hear (Ed.), Royal Institute of Philosophy Supplement (125th ed., Vol. 82, p. 154). London: Royal Institute of Philosophy. 